\title{
Formação de micorriza em variedades de oliveira (Olea europaea L.) com potencial de cultivo em Minas Gerais
}

\author{
Mycorrhiza formation on olive varieties (Olea europaea L.) fit for cultivation in \\ the state of Minas Gerais
}

\section{Rogério Melloni' ${ }^{I}$, Fabrício Rezende Salomão ${ }^{\text {II }}$, Luiz Fernando de Oliveira da Silva ${ }^{I I I}$, Denise de Lourdes Colombo Mescolotti ${ }^{\mathrm{IV}}$, Elke Jurandy Bran Nogueira Cardoso ${ }^{\mathrm{V}}$}

\begin{abstract}
Resumo
A oliveira, em razão da elevada demanda por produtos como azeitona e azeite de oliva, apresenta posição de destaque no mercado mundial de alimentos. Para o aprimoramento de seu cultivo, pesquisas relacionadas com fatores de ordem biológica da relação solo-planta estão ganhando enfoque, principalmente quanto ao uso de microrganismos como fungos micorrízicos arbusculares (FMAs). Contudo, há uma grande escassez de informação nessa área no Brasil, tanto em condições controladas quanto realizadas in situ. Nesse sentido, o presente trabalho visou avaliar a formação de propágulos (usando as características micélio extrarradicular, número e diversidade de esporos) de micorriza em 17 variedades de oliveira (Olea europaea L.). Essas variedades, com potencial de cultivo no estado de Minas Gerais, são mantidas no banco de germoplasma da Fazenda Experimental de Maria da Fé - EPAMIG (Empresa Brasileira de Pesquisa Agropecuária de Minas Gerais). Houve resposta distinta entre as variedades de oliveira para a formação de micélio extrarradicular e micorriza, sendo a colonização radicular in situ variada entre $0,4 \%$ e 3,6\%. Nas condições edafoclimáticas da região de pesquisa não foi detectada nenhuma influência das variedades de oliveira sobre o número total de esporos e a diversidade de FMAs, sendo que as espécies Glomus ambisporum e Acaulospora scrobiculata foram identificadas com maior frequência nas raízes das oliveiras analisadas.
\end{abstract}

Palavras-chave: Micorriza; Fungo micorrízicos arbuscular; Micélio; Olivicultura

\begin{abstract}
The olive tree, due to the high demand for the products olive and oil, is a relevant crop for the global food market. To improve its cultivation, increasing efforts have been invested in studies on biological factors of the soil-plant relationships, mainly with regard to the exploitation of microorganisms such as arbuscular mycorrhizal fungi (AMF). In Brazil however, further research is still needed, both under controlled and field conditions. In this sense, this study investigated the formation of extraradical mycelium and spore numbers and diversity of mycorrhiza, on 17 olive tree (Olea europaea L.) varieties. These varieties, considered promising for cultivation in the state of Minas Gerais, are maintained in the olive germplasm bank of the Agricultural Research Institute of Minas Gerais (EPAMIG), at the Experimental Farm Maria da Fé. The response of the olive varieties differed in terms of the level of extraradical mycelium and mycorrhiza formation, since root colonization in the field varied from $0.4 \%$ to $3.6 \%$. Under the edaphoclimatic conditions of the study region, no influence of the olive tree varieties on the total number of spores and diversity of AMF was detected, among which the fungal species Glomus ambisporum and Acaulospora scrobiculata were identified most frequently on the roots of the analyzed olive trees.
\end{abstract}

Keywords: Mycorrhiza; Arbuscular mycorrhizal fungus; Mycelium; Olive cultivation

Engenheiro Agrônomo, Dr., Professor do Instituto de Recursos Naturais, Universidade Federal de Itajubá, Av. BPS, 1303, Pinheirinho, CEP 37500903, Itajubá (MG), Brasil. rmelloni@unifei.edu.br (ORCID: 0000-0002-2724-2603)

II Engenheiro Agrônomo, Me., Pesquisador da empresa Nova Lima, Av. Coronel Carneiro Jr., N 142, CX 14, Bairro Centro, CEP 37500-018, Itajubá (MG), Brasil. fabriciorsagro@gmail.com (ORCID: 0000-0003-3314-8012)

III Engenheiro Agrônomo, Dr., Pesquisador da EPAMIG SUL, Rua Washington Alvarenga Viglione, s/n., Bairro Vargedo, CEP 37517-000, Maria da Fé (MG), Brasil. luizfernando.agronomia@gmail.com (ORCID: 0000-0002-4250-3621)

IV Tecnóloga em Gestão Ambiental, Técnica do Laboratório de Microbiologia do Solo, Departamento de Ciência do Solo, Escola Superior de Agricultura Luiz de Queiroz, Universidade de São Paulo, Av. Pádua Dias, 11, CEP 13418-900, Piracicaba (SP), Brasil. dlcmesco@usp.br (ORCID: 0000-0003-2449-3623)

V Engenheira Agrônoma, Dra., Professora do Departamento de Ciência do Solo, Escola Superior de Agricultura Luiz de Queiroz, Universidade de São Paulo, Av. Pádua Dias, 11, CEP 13418-900, Piracicaba (SP), Brasil. ejbncard@usp.br (ORCID: 0000-0003-4280-2514) 


\section{Introdução}

A oliveira (Olea europaea L.) é originária da parte oriental do Mediterrâneo, a qual compreende a região do Cáucaso até o Irã, Palestina e a zona costeira da Síria. Com a expansão das civilizações no Mediterrâneo, o cultivo de oliveiras difundiu-se nos países tradicionalmente reconhecidos como os principais produtores, como a Espanha, Grécia, Itália e outros (COUTINHO et al., 2009). Com o descobrimento da América, a olivicultura passou a ser cultivada em países que vão desde a Argentina e Chile até os Estados Unidos.

No Brasil, tem-se verificado aumento na produtividade de muitas culturas agrícolas, pela expansão na sua extensa área territorial. Por outro lado, a introdução de novas espécies economicamente produtivas de outros países vem se destacando no mercado agrícola nacional. A oliveira é um exemplo dessas novas espécies, sendo cultivada principalmente em regiões que atendem às condições climáticas necessárias ao seu desenvolvimento (PANTANO; BEERTONCINI; WREGE, 2014).

A formação de olivais comerciais no Brasil teve início na década passada, na Fazenda Experimental de Maria da Fé - FEMF, da Empresa Brasileira de Pesquisa Agropecuária de Minas Gerais - Epamig. Essa instituição tornou-se pioneira na extração de azeite de oliva do Brasil, no ano de 2008 (EPAMIG, 2018). Baseado em dados da safra de 2018, estima-se que existem 400 olivais, distribuídos em 70 municípios brasileiros, ocupando uma área plantada de aproximadamente 2.500 hectares, com produção total estimada de 80 mil litros (EPAMIG, 2018), valor considerado incipiente em relação ao total importado.

A produção brasileira de azeitona e óleo de oliva está longe de ser autossuficiente e, por ser uma cultura ainda recente, enfrenta fatores limitantes. As principais dificuldades encontradas pelos produtores têm sido alcançar preços sustentáveis para a produção de azeitonas, reduzir a sazonalidade entre safras, obter variedades mais adaptadas ao clima brasileiro e, com isso, contornar a limitação do zoneamento climático. Devido a esse fenômeno, os olivais estão concentrados em regiões de clima mais ameno, como nos estados do Rio Grande do Sul, Santa Catarina, Espírito Santo, Minas Gerais, Rio de Janeiro e São Paulo. Estudos realizados para o zoneamento da cultura em São Paulo indicam que o requerimento de frio para a produção de oliveiras é de exposição a 500 a 1.000 horas de temperaturas abaixo de $13^{\circ} \mathrm{C}$, por ano ou por safra, o que pode variar de acordo com cada microclima regional (PANTANO; BEERTONCINI; WREGE, 2014).

Pesquisas têm sido realizadas a fim de identificar fatores de ordem biológica que podem reduzir os efeitos negativos provocados pelo processo adaptativo das oliveiras no Brasil (SILVA et al., 2018). Uma das linhas de pesquisa que pode contribuir para um desenvolvimento melhor da cultura na lavoura são os estudos relacionados aos fungos micorrízicos arbusculares (FMAs). Esses fungos possuem comprovada capacidade de estimular o crescimento das plantas, uma vez que favorecem a absorção de nutrientes do solo. Além disso, propiciam a estabilização dos agregados na rizosfera, bem como a mitigação de efeitos provocados por estresses bióticos e abióticos, a exemplo do aumento da resistência ao ataque de patógenos e da redução do estresse hídrico (MATOS et al., 2018). Dessa forma, respostas positivas em diferentes níveis de utilização de FMAs foram constatadas em diferentes combinações de espécies vegetais micotróficas (CUI et al., 2018).

Os efeitos benéficos dos FMAs têm sido demonstrados nas variedades de oliveiras cultivadas em diferentes países. A estrutura e diversidade de FMAs na rizosfera de oliveiras cultivadas na Espanha foram analisadas por Montes-Borrego, Metsis e Landa (2014), em 96 olivais, empregando técnicas moleculares. Observou-se uma maior formação micorrízica em oliveiras das variedades Arbequina, Picual e Manzanilla, principalmente pelo gênero Glomus. Recomenda-se a inoculação de FMAs nas mudas de oliveira cultivadas em viveiro, como uma prática comum a ser implementada nos olivais, principalmente por favorecer o estabelecimento da planta em campo. 
Outra demonstração prática da importância desses fungos foi feita na Argentina por Bompadre et al. (2014), ao inocular o FMA Rhizophagus irregularis em mudas de oliveiras cultivadas, resultando em melhoria de seu desenvolvimento na fase de viveiro e facilitando o transplantio para o campo. Os autores Meddad-Hamza et al. (2017) também verificaram que os climas úmidos e subúmidos favorecem o desenvolvimento de alguns FMAs na rizosfera de oliveiras, o que permite a expansão dessa cultura em países que apresentam clima semelhante, como o Brasil.

Estudos que relacionem FMAs à oliveira são de extrema importância e devem ser estimulados, já que a interação entre plantas e microrganismos é fundamental para a sustentabilidade agrícola e escolha das variedades agrícolas a serem utilizadas deve garantir as condições para uma associação com os microrganismos que mais se correlacionam com estas, tornando-as, dessa forma, mais vigorosas e produtivas. Nesse sentido, o objetivo do presente trabalho foi avaliar a formação de propágulos e micorriza em diferentes variedades de oliveira com potencial de cultivo para o estado de Minas Gerais, Brasil.

\section{Material e método}

O banco de germoplasma (BAG) estudado pertence ao Núcleo Tecnológico Epamig Azeitona e Azeite, da Fazenda Experimental da Epamig (FEMF), no município de Maria da Fé (MG). Esse acervo de muitas das variedades de oliveira no Brasil representa uma coleção de ampla variabilidade genética da espécie, incluindo muitos genótipos de interesse agronômico. Segundo Silva et al. (2012), o BAG foi instalado em 2005, em solo do tipo Latossolo Vermelho, com textura média argilosa, com o plantio de 60 genótipos diferentes de oliveira, em espaçamento de $7 \times 5 \mathrm{~m}$, em uma área de $15.000 \mathrm{~m}^{2},\left(22^{\circ} 18^{\prime} 57.97^{\prime \prime} \mathrm{S}, 45^{\circ} 22^{\prime} 28.82^{\prime \prime} \mathrm{O}, 1.311 \mathrm{~m}\right.$ alt $)$. As plantas foram conduzidas de forma livre, sendo realizadas pequenas intervenções de poda, como a limpeza de ramos mal posicionados e/ou que apresentam algum problema fitossanitário. Além disso, com base em análises de solo e foliar, foram realizadas calagens a cada dois anos, de modo a manter o pH entre 6,0 e 6,5, e duas adubações anuais. A primeira foi aplicada após a colheita para restituir os nutrientes retirados pela mesma, e a segunda dividida em três adubações (em outubro, novembro e janeiro), visando fornecer nutrientes para o ciclo de produção seguinte.

Para este estudo, 17 variedades foram selecionadas, observando a representação de diferentes países de origem, o número suficiente de plantas presentes no BAG (mínimo de três por variedade) eo vigor atual dessas. As variedades e seus países de origem foram: Alto do D'Ouro (Espanha), Arbequina (Espanha), Arbosana (Portugal), Ascolana USA (Itália), Ascolana 315 (Itália), Barnea (Síria), Frantoio (Itália), Galega (Espanha), Grappolo (Itália), Grappolo 541 (Itália), Grappolo 575 (Itália), Koroneiki (Grécia), Leccino (Itália), Maria da Fé (Brasil), Mission (Estados Unidos), Negroa(Espanha) e Tafahi (Egito). Em seguida, procedeu-se à coleta de três amostras simples por planta de solo rizosféricoe das raízes, na profundidade de $0-20 \mathrm{~cm}$.

As amostras de solo, coletadas em maio de 2018, foram peneiradas em malha de $2 \mathrm{~mm}$ e armazenadas em câmara refrigerada, com temperatura média de $4^{\circ} \mathrm{C}$. O solo peneirado foi utilizado para análise do micélio extrarradicular total (MET)e da densidade de esporos de FMAs, enquanto as amostras das raízes foram conservadas em álcool 70\% para determinação posterior da colonização radicular.

Para determinara umidade das amostras, foram pesadas pequenas frações de solo em recipientes metálicos, antes e após secagem em estufa aquecida a $105^{\circ} \mathrm{C}$, por 24 horas (TEIXEIRA et al., 2017).Os resultados desse procedimento foram usados para estabelecer a unidade do micélio extrarradicular total (MET), a qual foi determinada conforme metodologia proposta por Melloni e Cardoso (1999), sem alterações, com observação e contagem em microscópio óptico com ocular reticulada.

A extração dos esporos das FMAs foi feita conforme procedimento descrito por Gerdemann e Nicolson (1963), utilizando $50 \mathrm{ml}$ de solo rizosférico, seguida de lavagens e centrifugações sucessivas, e contagem posterior dos esporos em placa concêntrica, com uso delupa. 
Para avaliação, os esporos foram distribuídos em lâminas de microscopia com PVLG (polivinil álcool-lactoglicerol) e PVLG/reagente de Melzer (4:1, v:v), antes da sua identificação conforme a descrição contida no sítio do INVAM (International Culture Collection of Vesicular Arbuscular Mycorrhizal Fungi) e cálculo da diversidade de Shannon.

Para a determinação da colonização radicular foi utilizado o protocolo de Vierheiliget al. (1998), nas dependências do Laboratório de Microbiologia do Solo, da Escola Superior de Agricultura Luiz de Queiroz (Esalq). As lâminas contendo os segmentos de raízes foram avaliadas em microscópio óptico, com aumento total de 100x, atribuindo-se notas de 0 a 100, conforme a ocupação radicular, a qual expressa a intensidade de colonização radicular pelos FMAs.

Da área de projeção da copa das oliveiras estudadas, foram coletadas, de forma complementar, 25 amostras simples de solo, na profundidade de 0 a $20 \mathrm{~cm}$, por meio de trado holandês, a fim de obter uma amostra composta. Esta, por sua vez, foi encaminhada para o Laboratório Agronômico da Unital (Campinas-SP), para a determinação de macro e micronutrientes, e também dos atributos granulometria, densidade aparente e densidade real (TEIXEIRA et al., 2017).

Para análise estatística, os dados microbiológicos foram submetidos inicialmente ao teste de Shapiro Wilk a 5\% de probabilidade, para verificar a distribuição normal das variâncias. Posteriormente, fez-se análise de variância (ANOVA) para avaliar a significância dos efeitos das variedades, por meio da comparação de médias por Scott-Knott a 5\% de probabilidade, utilizando o software Sisvar.

\section{Resultados e discussão}

\section{Caracterização química e física do solo}

O resultado e a interpretação das amostras de solo são apresentados na Tabela 1. Exceto para o micronutriente boro, a amostra representativa do solo do BAG apresentou bons níveis de macro e micronutrientes. Essa condição melhora a relação solo-planta, interferindo diretamente na formação de simbiose por microrganismos presentes na rizosfera, como os FMAs (MOREIRA; SIQUEIRA, 2006; CARDOSO et al., 2010).

Os dados das análises das amostras validam as ações antrópicas de correção e adubação do solo, principalmente quanto aos níveis de fósforo, potássio e de $\mathrm{pH}$. É importante destacar que tais intervenções em áreas agrícolas podem desencadear efeitos significativos no desenvolvimento de microrganismos, principalmente quando comparados a áreas sem essas interferências (RAMOS et al., 2012).

Nesse sentido, condições elevadas de fertilidade, principalmente um alto nível de $\mathrm{P}$, podem inibir a formação micorrízica, enquanto a baixa fertilidade favorece a simbiose dos FMAs com seus hospedeiros (CARDOSO et al., 2010). A concentração de P no solo do presente trabalho encontra-se na faixa considerada ideal de fertilidade para as plantas. No entanto, altos níveis dos micronutrientes $\mathrm{Zn}$ e $\mathrm{Cu}$ podem afetar diretamente os propágulos de FMAs, interferindo no processo de micorrização em virtude da sua elevada sensibilidade a esses elementos (TRINDADE; SIQUEIRA; ALMEIDA, 2000).

Embora os FMA tolerem uma ampla faixa de pH no solo (3 a 10) (MOREIRA; SIQUEIRA, 2006) o valor mais favorável pode variar bastante de acordo com as espécies presentes no local. O valor de 5,5 , encontrado no lugar de cultivo pode promover a formação micorrízica de algumas espécies de Glomus, mas não de todas, sendo mais adequada para outros gêneros como Acaulospora, Gigaspora e Scutellospora e outras (LAMBAIS; CARDOSO, 1988; CAMARA et al., 2016). A diversidade de FMAs observada no presente estudo será detalhada posteriormente.

O solo da área de estudo foi caracterizado como argiloso, com $58 \%$ de argila. No entanto, segundo Coutinho et al. (2009), a oliveira se desenvolve melhor em solos de textura média (COUTINHO et al., 2009), com teor de argila menor que $15 \%$ e boa drenagem. 
Os valores de umidade nas amostras de solo variaram entre 15 e $24 \%$, evidenciando baixa variação e disponibilidade de água no solo. Isso pode ser explicado pela homogeneidade das práticas de cultivo em toda a área e a queda na precipitação durante o período de coleta das amostras, realizada no mês de maio de 2018.

Tabela 1 - Resultados e interpretação dos atributos químicos e físicos do solo

Table 1 - Results and interpretation of soil physical and chemical properties

\begin{tabular}{|c|c|c|c|}
\hline & Resultado & Unidades & Interpretação* \\
\hline C & 2,1 & $\mathrm{dag} / \mathrm{kg}$ & Médio \\
\hline MO & 3,6 & $\mathrm{dag} / \mathrm{kg}$ & Médio \\
\hline SB & 5,39 & $\mathrm{cmol} / \mathrm{c}^{3}$ & Bom \\
\hline $\mathbf{T}$ & 9,39 & $\mathrm{cmol} / \mathrm{cdm}^{3}$ & Bom \\
\hline $\mathbf{V}$ & 57,40 & $\%$ & Médio \\
\hline pH & 5,5 & em água & Médio \\
\hline \multicolumn{4}{|l|}{ Macronutrientes: } \\
\hline $\mathbf{P}$ & 52 & $\mathrm{mg} / \mathrm{dm}^{3}$ & Muito bom \\
\hline K & 113,39 & $\mathrm{mg} / \mathrm{dm}^{3}$ & Bom \\
\hline $\mathrm{Ca}$ & 4,2 & $\mathrm{cmol}_{\mathrm{c}} / \mathrm{dm}^{3}$ & Muito bom \\
\hline $\mathrm{Mg}$ & 0,9 & $\mathrm{cmol}_{\mathrm{c}} / \mathrm{dm}^{3}$ & Muito bom \\
\hline \multicolumn{4}{|l|}{ Micronutrientes: } \\
\hline$S$ & 14,2 & $\mathrm{mg} / \mathrm{dm}^{3}$ & Muito bom \\
\hline B & 0,3 & $\mathrm{mg} / \mathrm{dm}^{3}$ & Muito baixo \\
\hline $\mathrm{Fe}$ & 42,0 & $\mathrm{mg} / \mathrm{dm}^{3}$ & Bom \\
\hline Mn & 7,5 & $\mathrm{mg} / \mathrm{dm}^{3}$ & Médio \\
\hline $\mathrm{Cu}$ & 9,5 & $\mathrm{mg} / \mathrm{dm}^{3}$ & Alto \\
\hline $\mathrm{Zn}$ & 12,0 & $\mathrm{mg} / \mathrm{dm}^{3}$ & Alto \\
\hline \multicolumn{4}{|l|}{ Granulometria: } \\
\hline Cascalho & 0 & $\%$ & \\
\hline Areia grossa & 11,8 & $\%$ & \\
\hline Areia fina & 8,5 & $\%$ & \\
\hline Argila & 58,3 & $\%$ & \\
\hline Silte & 21,4 & $\%$ & \\
\hline Densidade aparente & 1,0 & $\mathrm{~kg} / \mathrm{dm}^{3}$ & \\
\hline Densidade real & 3,1 & $\mathrm{~kg} / \mathrm{dm}^{3}$ & \\
\hline Classe textural & Argila & - & Argilosa \\
\hline
\end{tabular}

Fonte: Autores (2019)

Em que: * Interpretação de acordo com CFSEMG (1999) e Novais, Smith e Nunes (2007).

A variação da umidade no solo tem influência na produção de propágulos e na colonização radicular por FMAs, sendo que estes se desenvolvem melhor em períodos chuvosos, com maior umidade 
no solo (MOREIRA; SIQUEIRA, 2006). Nesse sentido, Guadarrama e Alvarez-Sánchez (1999) explicaram que a umidade favorece a germinação dos esporos, proporcionando maior colonização. Ainda, podese dizer que, apesar do crescimento das raízes ocorrer normalmente ao longo do ano, a variação da quantidade de água e nutrientes pode interferir no comprimento radicular e na formação de micorriza.

\section{Formação de propágulos e micorriza}

O efeito das variedades de oliveira na formação de propágulos de FMAs e de micorriza pode ser visto na Tabela 2.

Não foi detectado um efeito significativo das variedades de oliveira sobre o número de esporos formados na rizosfera (Tabela 2), apesar da ampla faixa observada e resultados chegando a 80 esporos $/ 50 \mathrm{~g}$ de solo rizosférico, como observado para a variedade Koroneiki. Em outro estudo in situ, Vieira et al. (2011) também registraram baixa densidade de esporos na rizosfera de sete variedades de oliveiras, variando de 12 a 30 esporos por 50g de solo para as variedades Arbequina e Ascolano 315, respectivamente.

Tabela 2 - Efeito das variedades de oliveira na formação de propágulos (número de esporos, diversidade de Shannon e comprimento de micélio extrarradicular total) e de micorriza (colonização radicular)

Table 2 - Effect of olive varieties on the formation of propagules (number of spores, Shannon diversity index and total extraradical mycelium length) and mycorrhiza (root colonization)

\begin{tabular}{|c|c|c|c|c|}
\hline $\begin{array}{l}\text { Variedade } \\
\text { de oliveira }\end{array}$ & $\begin{array}{c}\text { Número de esporos } / 50 \mathrm{~g} \text { de } \\
\text { solo rizosférico }\end{array}$ & $\begin{array}{l}\text { Diversidade } \\
\text { de Shannon }\end{array}$ & $\begin{array}{l}\text { Micélio extrarradicular total } \\
\text { (m/g de solo rizosférico) }\end{array}$ & $\begin{array}{l}\text { Colonização } \\
\text { radicular (\%) }\end{array}$ \\
\hline Alto do D'Ouro & 30,0 & 0,81 & $3,52 b$ & $2,07 \mathrm{a}$ \\
\hline Arbequina & 28,3 & 0,74 & $2,68 \mathrm{c}$ & $0,77 \mathrm{~b}$ \\
\hline Arbosana & 32,6 & 1,02 & $2,81 \mathrm{c}$ & $2,20 \mathrm{a}$ \\
\hline Ascolana USA & 22,0 & 0,54 & $3,76 b$ & $3,63 a$ \\
\hline Ascolana 315 & 34,0 & 0,47 & $5,51 \mathrm{a}$ & $2,30 \mathrm{a}$ \\
\hline Barnea & 37,6 & 0,75 & $1,96 \mathrm{c}$ & $0,47 \mathrm{~b}$ \\
\hline Frantoio & 37,0 & 0,90 & $3,59 b$ & $0,93 b$ \\
\hline Galega & 49,3 & 0,94 & $2,30 \mathrm{c}$ & $1,00 \mathrm{~b}$ \\
\hline Grappolo & 31,6 & 0,92 & $2,99 \mathrm{c}$ & $1,81 \mathrm{a}$ \\
\hline Grappolo 541 & 13,6 & 0,72 & $2,67 \mathrm{c}$ & $0,80 \mathrm{~b}$ \\
\hline Grappolo 575 & 17,3 & 0,56 & $0,83 \mathrm{c}$ & $0,87 b$ \\
\hline Koroneiki & 80,3 & 0,67 & $4,02 \mathrm{~b}$ & $0,88 b$ \\
\hline Leccino & 28,6 & 0,59 & $4,30 \mathrm{~b}$ & $1,58 \mathrm{a}$ \\
\hline Maria da Fé & 67,6 & 0,98 & $3,29 b$ & $1,11 \mathrm{~b}$ \\
\hline Mission & 78,3 & 0,97 & $3,78 b$ & $1,74 \mathrm{a}$ \\
\hline Negroa & 33,3 & 0,27 & $2,99 \mathrm{c}$ & $0,48 b$ \\
\hline Tafahi & 25,6 & 0,76 & $2,35 c$ & $0,43 \mathrm{~b}$ \\
\hline CV (\%) & $35 \%$ & $32 \%$ & $25 \%$ & $33 \%$ \\
\hline
\end{tabular}

Fonte: Autores (2020)

Em que: CV - Coeficiente de variação. Médias seguidas por letras iguais não diferem estatisticamente entre si pelo teste de Scott-Knott, a 5\% de significância. 
Os esporos isolados e identificados da rizosfera das variedades avaliadas neste estudo pertencem a cinco espécies, e estas a três gêneros: Glomus, Acaulospora e Gigaspora (Figura 1).

Figura 1 - Esporos de fungos micorrízicos arbusculares isolados da rizosfera das variedades de oliveira: A) Glomus glomerulatum; B) Glomus ambisporum; C) Acaulospora scrobiculata; D) Acaulospora denticulata; E) Gigaspora sp

Figure 1 - Spores of arbuscular mycorrhizal fungi isolated from ther hizosphere of the olive varieties: A) Glomus glomerulatum; B) Glomus ambisporum; C) Acaulospora scrobiculata; D) Acaulospora denticulata; E) Gigaspora sp

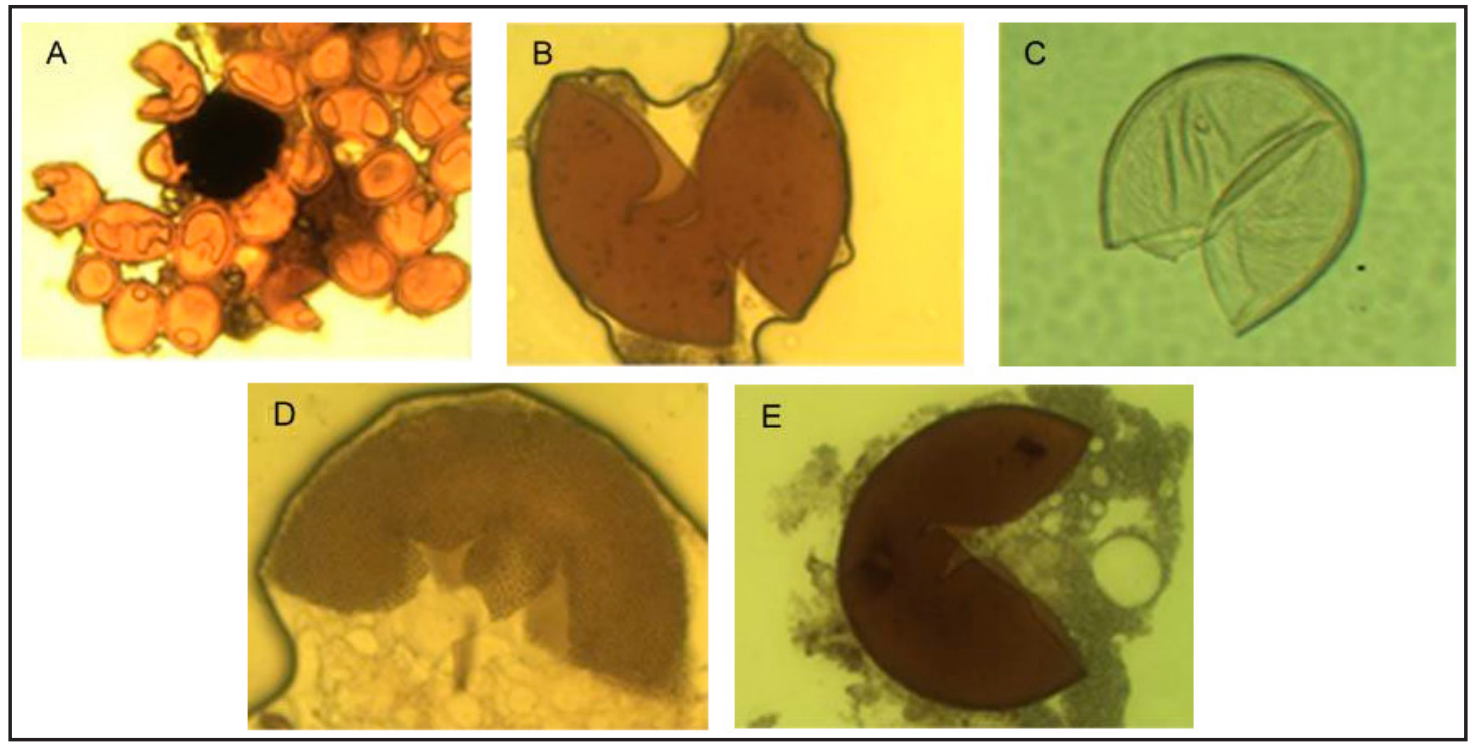

Fonte: Autores (2019)

A distribuição das espécies de FMA na rizosfera das variedades de oliveira pode ser vista na Figura 2. Glomus ambisporum e Acaulospora scrobiculata foram encontradas em todas as amostras, com maior domínio da primeira espécie, como também relatado por Vieira, Melloni e Vieira Neto (2011), no mesmo BAG, com outras variedades. Já a Acaulospora denticulata foi observada apenas nas variedades Arbosana, Ascolana 315 e Grappolo 575. Gigaspora sp., por sua vez, foi encontrada em menor frequência em quase todas as variedades estudadas, exceto na Ascolana USA, Grappolo 575 e Tafahi.

O gênero Glomus tem sido frequentemente encontrado em levantamentos sobre a colonização micorrízica na rizosfera de oliveiras feitos em outros países, como Marrocos (KACHKOUCH et al., 2012). No entanto, outros gêneros têm sido relatados por Kachkouch et al. (2014), como Gigaspora, Acaulospora e Entrophospora. No presente estudo, não houve efeito das variedades de oliveira na diversidade de esporos de FMAs, com valor médio de 0,75 (Tabela 2). Baixa diversidade também foi relatada por Vieira, Melloni e Vieira Neto (2011), em outro estudo em campo, com outras variedades. Esses últimos autores salientaram que o estudo da diversidade de FMAs na rizosfera de oliveiras representa um primeiro passo no desenvolvimento de inoculantes adequados para a produção de mudas, buscando a obtenção de plantas mais vigorosas e resistentes a patógenos e ao estresse hídrico após o transplantio.

Em relação à quantidade de micélio extrarradicular total (MET), houve efeito significativo das variedades (Tabela 2), com a formação de três grandes grupos, sendo: grupo I, representado pela variedade Ascolano 315 (5,51 $\mathrm{m} \mathrm{g}^{-1}$ de solo seco); grupo II, com as variedades Alto do Ouro, Ascolano USA, Frantoio, Koroneiki, Leccino, Maria da Fé e Mission (valores de 3,29 a 4,30 m 
$\mathrm{g}^{-1}$ de solo seco); e grupo III, composto das demais variedades (de 0,83 a $2,99 \mathrm{~m} \mathrm{~g}^{-1}$ de solo seco). O maior comprimento do MET observado na rizosfera da Ascolano 315 pode indicar maior dependência da associação micorrízica para o desenvolvimento dessa variedade de oliveira, conforme sugerido por Balota et al. (1997), ao descreverem esse resultado para outra espécie vegetal, Manihot esculenta.

\section{Figura 2 - Porcentagem de distribuição das espécies de fungos micorrízicos arbusculares} (FMAs) na rizosfera das diferentes variedades de oliveira

Figure 2 - Percentage distribution of AMF species in the rhizosphere of different olive varieties

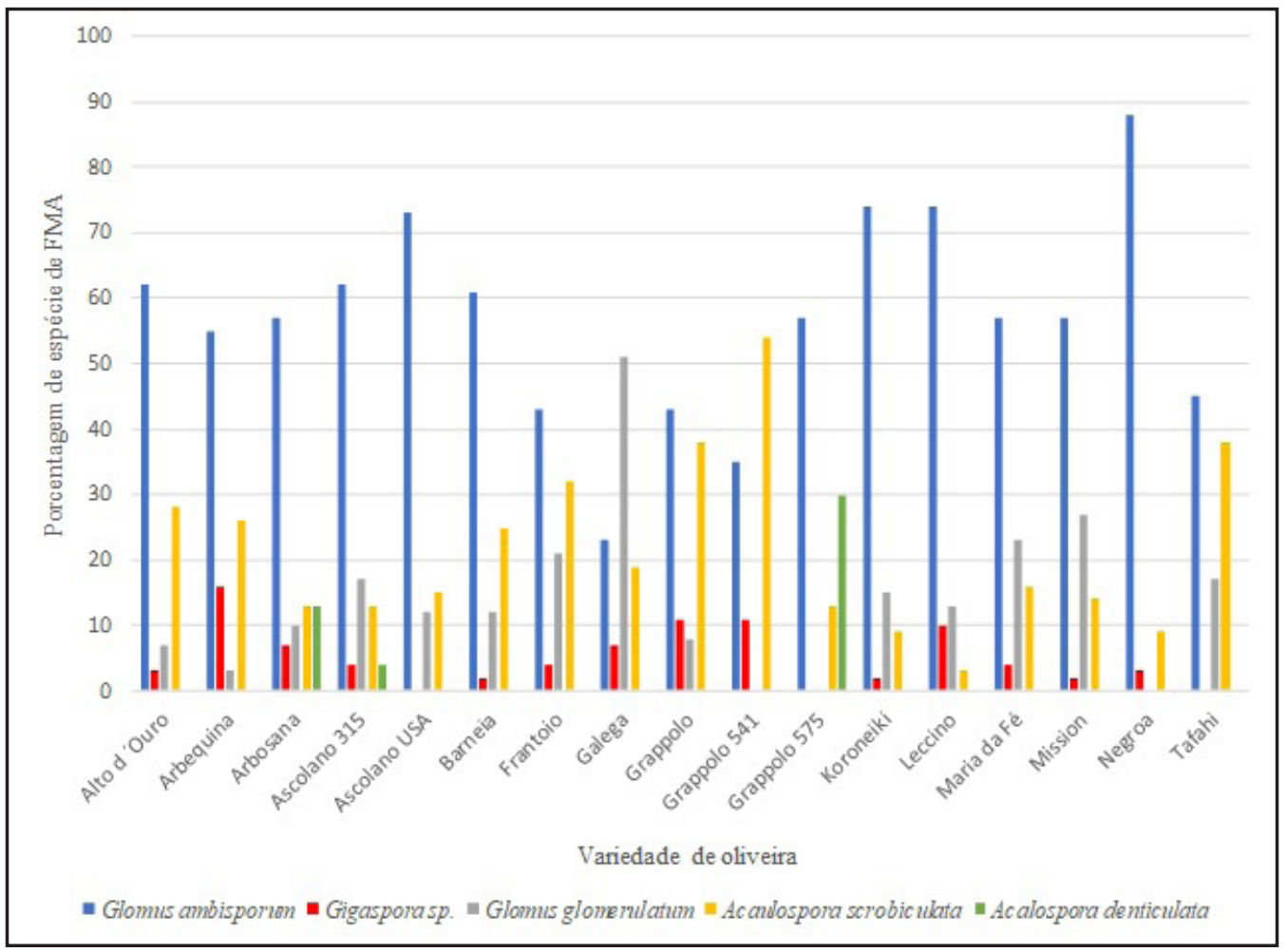

Fonte: Autores (2020)

Em estudo in situ conduzido por Vieira, Melloni e Vieira Neto (2011), as variedades de oliveira não influenciaram o comprimento de MET, com valores inferiores, na ordem de 2,4 a $3,7 \mathrm{mg}^{-1}$ de solo seco. Na referida pesquisa, a amostragem foi realizada ao final do verão, em março, enquanto as amostras deste estudo foram coletadas em maio. A variação do comprimento de MET relatada nesses trabalhos pode ter sido consequência do efeito das diferenças climáticas, já que no verão a temperatura e precipitação são mais altas, influenciando no maior crescimento radicular e, consequentemente, menor necessidade de formação de micélio, ou ainda, na maior predação de micélio por outros microrganismos no solo nessa época (MOREIRA; SIQUEIRA, 2006).

Houve efeito significativo, também, das variedades de oliveira para colonização radicular in situ, a qual variou de $0,4 \%$ a 3,6\% (Tabela 2), com a formação de dois grandes grupos, representados pela Tafahi e Ascolano USA, extremos de menor e maior valor, respectivamente.

De modo geral, a formação micorrízica é favorecida quando se observa baixa disponibilidade de nutrientes, especialmente baixos teores de $\mathrm{P}$, que favorecem a ação desses fungos no sistema solo-planta (FEITOSA; SANTOS, 2016). Essa correlação também foi observada no presente 
trabalho, verificando baixa colonização radicular, uma vez que a área estudada recebeu insumos agrícolas, o que aumenta a fertilidade do solo (Tabela 1). Da mesma forma, tem sido relatada a baixa colonização in situ, em contraste com aqueles conduzidos em condições controladas em casa de vegetação (VIEIRA; MELLONI; VIEIRA NETO, 2011), em concordância com os valores de colonização radicular nos estudos de Castilho et al. (2006), entre $78 \%$ e $95 \%$, com o efeito da inoculação de FMAs Glomus mosseae, G. intraradices e G. viscosum nas variedades Arbequina e Picual. Por outro lado, no estudo de Costa e Melloni (2019), foram inoculadas três espécies de FMAs (Acaulospora scrobiculata, Gigaspora rosea e Rhizophagus clarus) e verificou-se colonização na ordem de 11 a 22\% na Arbequina, Maria da Fé e Grappolo 541, promovendo, mesmo assim, efeito significativo no crescimento das mudas dessas variedades.

Os estudos da relação entre as variedades de oliveira e a micorrização obviamente são afetadas pela interferência das práticas agrícolas como adubação e correção de acidez no solo, constatando-se que os resultados obtidos em condições controladas diferentes podem não ser semelhantes. Mesmo assim, a prática da indução da formação de propágulos de FMAs é indicada como estratégia para a promoção de maior adaptabilidade e sobrevivência das plantas de oliveira.

\section{Conclusão}

Nas mesmas condições edafoclimáticas, as variedades de oliveira apresentavam variações na formação de micorriza e no comprimento de micélio extrarradicular total, com maiores porcentagens encontradas para as variedades Alto d'Ouro, Arbosana, Ascolano 315, Ascolano USA, Grappolo, Leccino e Mission.

Nas condições estudadas no local de pesquisa, não foi detectada nenhuma influência das variedades de oliveira sobre o número total de esporos e a diversidade de FMAs, sendo que as espécies Glomus ambisporum e Acaulospora scrobiculata foram identificadas com maior frequência nas raízes das oliveiras analisadas.

\section{Agradecimentos}

À Empresa de Pesquisa Agropecuária de Minas Gerais (Epamig), Fundação de Amparo à Pesquisa de Minas Gerais (Fapemig), Escola Superior de Agricultura Luiz de Queiroz (Esalq/ USP) e Coordenação de Aperfeiçoamento de Pessoal de Nível Superior (Capes), pelo apoio.

\section{Referências}

BALOTA, E. L.; LOPES, E. S.; HUNGRIA, M.; DOBEREINER, J. Inoculação de bactérias diazotróficas e fungos micorrízicos arbusculares na cultura da mandioca. Pesquisa Agropecuária Brasileira, Brasília, v. 32, p. 627-639, 1997.

BOMPADRE, M. J.; PÉRGOLA, M.; FERNÁNDEZ BIDONDO, L.; COLOMBO, R. P.; SILVANI, V. A.; PARDO, A. G.; CAMPO, J. A.; GODEAS, A. M. Evaluation of arbuscular mycorrhizal fungi capacity to all eviate abiotic stress of olive (Olea europaea L.) plants at diferente transplant conditions. The Scientific World Journal, London, v. 1, p. 1-13, 2014.

CAMARA, R.; PEREIRA, M. G.; RANIERI, C. F. S.;PAULA, R. R.; SILVA, E. M. R. Fungos micorrízicos arbusculares em dois fragmentos florestais de restinga periodicamente inundável em Marambaia - RJ. Floresta e Ambiente, Seropédica, v. 23, p. 33-42, 2016.

CARDoso, J. B. N.; CARDoso, I. M.; NOGUEIRA, M. A.; BARETTA, C. R. D. M.; PAUlA, 
A. M. Micorrizas arbusculares na aquisição de nutrientes pelas plantas. In: SIQUEIRA, J. O.; SOUZA, F. A.; CARDOSO, E. J. B. N.; TSAI, S. M. Micorrizas: 30 anos de pesquisas no Brasil. Lavras: UFLA, 2010. cap. 6. p. 153-214.

CASTILHO, P.; NICO, A. L.; AZCÓN-AGUILAR, C.; CALVET, C.; JiMÉNEZ-DÍAZ, R. M. Protection of olive planting stocks against parasitism of root-knot nematodes by arbuscular mycorrhizal fungi. Plant Pathology, Seoul, v. 55, p. 705-713, 2006.

CFSEMG - Comissão de Fertilidade do Solo do Estado de Minas Gerais. Recomendações para o uso de corretivos e fertilizantes em Minas Gerais: 5ª aproximação. Viçosa: UFV, 1999.

COSTA, S. M.; MELLONI, R. Relação de fungos micorrízicos arbusculares e rizobactérias no crescimento de mudas de oliveira (Olea europaea). Ciência Florestal, Santa Maria, v. 29, n. 1, p. 169-180, 2019.

COUTINHO, E. F.; WREGE, M. S.; REISSER JÚNIOR, C.; ALMEIDA, I. R.; STEINMETZ, S. Cultivo de oliveira (Olea europaea L.): clima. Pelotas: Embrapa Clima Temperado, 2009.

CUI, J.; BAI, L.; LIU, X.; JIE, W.; CAI, B. Arbuscular mycorrhizal fungal communities in the rhizosphere of a continuous cropping soybean system at the seedling stage. Brazilian Journal of Microbiology, Amsterdam, v. 49, p. 240-247, 2018.

EPAMIG. Primeira extração do azeite brasileiro completa 10 anos. Comunicação da Empresa Brasileira de Pesquisa Agropecuária de Minas Gerais. Disponível em: https://epamig.wordpress. com/2018/03/26/primeira-extracao-do-azeite-brasileiro-completa-10-anos. Acesso em: 19 maio 2018.

FEITOSA, A. G.; SANTOS, D. R. Fósforo e fungos micorrízicos no crescimento de mundas arbóreas da caatinga. Agropecuária Científica no Semiárido, Campina Grande, v. 12, p. 392396, 2016.

GERDEMANN, J. W.; NICOLSON, T. H. Spores of mycorrhizal Endogone species extracted from soil by wet sieving and decanting. Transactions of British Mycological Society, Amsterdam, v. 46, p. 235-244, 1963.

GUADARRAMA, P.; ÁLVAREZ-SÁNCHEZ, F. J. Abundance of arbuscular mycorrhizal fungi spores in different environments in a tropical rain Forest, Veracruz, Mexico. Mycorrhiza, Berlin, v. 8, p. 267-270, 1999.

KACHKOUCH, W.; TOUATI, J.; TOUHAMI, A. O.; FILALI-MALTOUF, A.; MODAFAR, C. E.; MOUKHLI, A.; OUKABLI, A.; BENKIRANE, R.; DOUIRA, A. Diversity of arbuscular mycorrhizal fungi in the rhizosphere of Olea europaea in three regions of Morocco (Tafilalt, Zagora and Taounate). International Journal of Pure \& Applied Bioscience, Rajasthan, v. 2, p. 178-195, 2014.

KACHKOUCH, W.; TOUHAMI A. O.; FILALI-MALTOUF, A.; MODAFAR, C. E.; MOUKHLI, A.; OUKABLI, A.; BENKIRANE, R.; DOUIRA, A. Arbuscular mycorrhizal fungi species associated with rhizosphere of Olea europaea L. in Morocco. Journal of Animal \& Plant Sciences, Nairobi, v. 15, p. 2275-2287, 2012.

LAMBAIS, M.R.; CARDOSO, E.J.B.N. Avaliação da germinação de esporos de fungos micorrízicos vesículo-arbusculares e da colonização micorrízica de Stylosanthes guianensis em solo ácido e distrófico. R. Bras. Ci. Solo, 12:249-255, 1988.

MATOS, E. C. T.; RODRIGUES, L. A.; SOUZA, P. A.; SILVA, R.V.; FARIA JUNIOR, R. T. Espectroscopia fotoacústica para analisar a fertilidade de solos tratados com biochar e micorriza. Química Nova, São Paulo, v. 41, p. 989-998, 2018.

MEDDAD-HAMZA, A.; HAMZA, N.; NEFFAR, S.; BEDDIAR, A.; GIANINAZZI, S.; CHENCHOUNI, H. Spatiotemporal variation of arbuscular mycorrhizal fungal colonization 
in olive (Olea europaea L.) roots across a broad mesic-xeric climatic gradient in North Africa. Science of theTotal Environment, Amsterdam, v. 583, p. 176-189, 2017.

MELLONI, R.; CARDOSO, E. J. B. N. Quantificação de micélio extrarradicular de fungos micorrízicos arbusculares em plantas cítricas - I: método empregado. Revista Brasileira de Ciência do Solo, Viçosa, v. 23, p. 53- 58, 1999.

MONTES-BORREGO, M.; METSIS, M.; LANDA, B. B. Arbuscular Mycorhizal Fungi Associated with the Olive Crop across the Andalusian Landscape: Factors Community Differentiation. PlosOne, São Francisco, v. 9, p. 1-12, 2014.

MOREIRA F. M. S.; SIQUEIRA J. O. Microbiologia e bioquímica do solo. Lavras: UFLA. 2006. $729 \mathrm{p}$.

NOVAIS, R.F.; SMITH T. J.; NUNES F. N. Fósforo. In: Novais R.F., Alvares V.H., Barros N.F., Fontes R.B.C., Neves J.C.L., Fertilidade do solo. Sociedade Brasileira de Ciência do solo, Viçosa, p. 471-550, 2007.

PANTANO, A. P.; BEERTONCINI, E. I.; WREGE, M. S. Pré-zoneamento para a cultura da oliveira no Estado de São Paulo. O Agronômico, Campinas, v. 64/66, p. 52-55, 2014.

RAMOS, M. L. G.; KONRAD, M. L. F.; SILVA, D. E.; RIBEIRO JUNIOR, W. Q.; BATISTA, L. M. T. Diversidade de fungos micorrízicos e colonização radicular, em forrageiras solteiras e em consórcio com milho. Bioscience Journal, Uberlândia, v. 28, p. 235-244, 2012.

SILVA, P. B.; GIONGO, V.; LIMA JUNIOR, C., LIMA, R. L. F. A. Micorrizas arbusculares e teor de fósforo no solo em agroecossistemas de meloeiro no Semiárido. In: JORNADA DE INTEGRAÇÃO DA PÓS-GRADUAÇÃO DA EMBRAPA SEMIÁRIDO, 3., 2018, Petrolina. Anais... Petrolina: Embrapa Semiárido, 2018. p. 79-84. v. 3.

SILVA, L. F. O.; OLIVEIRA, A. F.; PIO, R.; ZAMBON, C. R. Caracterização agronômica e carpométrica de cultivares de oliveira. Pesquisa Agropecuária Tropical, Goiânia, v. 42, p. 350356, 2012.

TEIXeira, P. C.; DOnAGemma, G. K.; FOnTANA, A.; TEIXEIRA, W. G. Manual de métodos de análise de solo. - 3. ed. - Brasília: Embrapa, p. 573, 2017.

TRINDADE, A. V.; SIQUEIRA J. O.; ALMEIDA, F.P. Eficiência simbiótica de fungos micorrízicos arbusculares em solo não fumigado, para mamoeiro. Revista Brasileira de Ciência do Solo, Viçosa, v. 24, p. 505-513, 2000.

VIEIRA, V. C. S.; MELLONI, R.; VIEIRA NETO, J. Avaliação da interação micorrízica em variedades de oliveira (Olea europea L.). Revista Brasileira de Ciências do Solo, Viçosa, v. 35, p. 1885-1892, 2011.

VIERHEILIG, H.; COUGHLAN, A. P.; WYSS, U.; PICHÉ, Y. Ink and vinegar, a simple staining technique for arbuscular-mycorrhizal fungi. Applied and Environmental Microbiology, [s.l.], v. 64, p. 5004-5007, 1998. 